\title{
Compartilhamento do conhecimento: um estudo de caso no Instituto Federal de Santa Catarina
}

\author{
Knowledge sharing: case study of the Instituto Federal de Santa Catarina
}

\author{
Lenir Terezinha Zanuzzo Especialista, Universidade do Sul de Santa Catarina (UNISUL), Brasil - lenir.zanuzzo@gmail.com \\ Jaqueline Besen Especialista, Universidade do Sul de Santa Catarina (UNISUL), Brasil - jaque.besen@gmail.com \\ Fabiana Besen Santos Doutora, Instituto Federal de Santa Catarina (IFSC), Brasil - besen@ifsc.edu.br \\ Clarissa Carneiro Mussi Doutora, Universidade do Sul de Santa Catarina (UNISUL), Brasil - clarissa.mussi@unisul.br \\ Maurício Andrade de Lima Doutor, Universidade do Sul de Santa Catarina (UNISUL), Brasil - mauricio.lima@unisul.br
}

\section{RESUMO}

A gestão do conhecimento é um fenômeno complexo e vem estabelecendo uma nova dinâmica na forma como as organizações compartilham o conhecimento, em especial as organizações públicas. As instituições públicas buscam o aprimoramento das ferramentas de gestão do conhecimento, com vistas ao cumprimento de sua missão, visão e ao alcance de seus objetivos estratégicos. Compreender como ocorre o compartilhamento do conhecimento em uma instituição pública de ensino constitui-se o objetivo deste estudo. Para isso, o trabalho investigou o uso de ferramentas de compartilhamento de conhecimento no Instituto Federal de Santa Catarina (IFSC), por meio de análise documental e entrevista. Os resultados indicam que o compartilhamento de conhecimento se efetiva por meio de diferentes formas, das quais se destaca o uso do e-mail institucional, a realização de reuniões presenciais e não presenciais, a disponibilização de documentos em plataformas institucionais, a promoção de capacitações e o uso de aplicativo de mensagem instantânea. Estas ferramentas contribuem para a promoção da cultura de cooperação e união, favorecendo as relações entre os servidores que, por sua vez, desenvolvem seu trabalho com mais eficácia.

Palavras-chave: Gestão do conhecimento. Compartilhamento do conhecimento. Instituições públicas.

\begin{abstract}
Knowledge management is a complex phenomenon and has been setting new dynamics in the way organizations share knowledge, especially public organizations. Public institutions seek to improve knowledge management tools in order to fulfill their mission, vision and the achievement of their strategic objectives. Understanding how the sharing of knowledge occurs in a public institution of education is the purpose of this study. For this, this research investigated the use of knowledge sharing tools at the Federal Institute of Santa Catarina (IFSC), through documentary analysis and interview. The results indicate that knowledge sharing happens in different forms, such as the use of institutional e-mail, the holding of in-person and online meetings, the provision of documents on institutional platforms, the promotion of training and the use of instant messaging application. These tools contribute to the promotion of a culture of cooperation and unity, favoring relations between the servants who, in turn, develop their work more effectively.
\end{abstract}

Keywords: Knowledge management. Knowledge sharing. Public institutions. 


\section{INTRODUÇÃO}

O ambiente em que as organizações estão inseridas é caracterizado por mudanças rápidas e de múltiplas dimensões. Novos conhecimentos precisam ser criados continuamente para que a empresa sobreviva no ambiente competitivo atual (TAKEUCHI; NONAKA, 2008; HERRERA, 2008). De acordo com Drucker (1993), a cada dois ou três séculos ocorre uma grande transformação na história da sociedade ocidental, ela se reorganiza, passa por uma reformulação da visão de mundo, dos valores básicos, das estruturas sociais e políticas, das artes e das instituições mais importantes que a compõem.

As transformações que vêm ocorrendo, atualmente, colocam o conhecimento como o mais importante fator de produção. As atividades que ocupam o lugar central nas organizações da sociedade do conhecimento não são mais aquelas que visam produzir ou distribuir objetos, mas sim aquelas que usam o conhecimento como elemento principal para geração de riqueza (DRUCKER, 1993; VON KROGH, 2012; ZHANG et al., 2015)

A importância do conhecimento para a economia mundial é inegável (DRUCKER, 1993; OCDE, 1997). No entanto, o entendimento do que é conhecimento apresenta variações na literatura. Uma definição clássica, proposta por Platão, aponta que conhecimento é uma crença verdadeira e justificada. Nonaka e Takeuchi (1997) seguem essa linha de pensamento e definem o conhecimento como um processo humano dinâmico de justificar a crença pessoal com relação à verdade.

O conhecimento é mais rico que a informação, com mais significado e é uma forma das organizações produzirem riquezas (SVEIBY, 2005; ZABOT; SILVA, 2002). Contudo, cabe ressaltar, que a informação é um meio necessário para a extração e construção do conhecimento (TAKEUCHI; NONAKA, 2008). A informação é transformada no ambiente organizacional, gerando a capacidade de adaptação, aprendizagem e inovação (BARBOSA, 2008).

Este estudo adotará o conceito de Bhatt (2001, p. 4), que afirma que "o conhecimento é uma combinação organizada de dados, assimilados com um conjunto de regras, procedimentos e operações aprendidas através da experiência e da prática."

Assim, de um lado tem-se que o conhecimento se consolida como um recurso estratégico fundamental para a manutenção da vantagem competitiva das organizações (MAXIMIANO, 2004; TERRA, 2005; DAVENPORT, PRUSAK, 1998). Por outro, temos a questão de como gerir um elemento intangível, difícil de ser mensurado.

A Gestão do Conhecimento (GC) é uma atividade complexa e repleta de desafios. Apesar de ser um tema recente no mundo acadêmico, discutido mais intensamente na década de 90 , identificam-se diversos conceitos e modelos. Dentre as proposições da literatura, a GC é definida como um processo que promove o fluxo do conhecimento, constituído por algumas atividades essenciais: criação/aquisição, armazenamento, compartilhamento/distribuição e utilização do conhecimento (DURST; EDVARDSSON, 2012; LIAO, CHUANG, TO, 2011; ARGOTE, MCEVILY, REAGANS, 2003). As organizações capazes de desenvolver essas atividades da GC de forma eficaz se destacam e mantêm uma vantagem sustentável (DAVENPORT; PRUSAK, 1998; SALIM, 2003).

Dentre as etapas da GC, o compartilhamento ou distribuição é o foco deste artigo. O compartilhamento consiste na difusão do conhecimento criado ou adquirido para os demais indivíduos da organização (LEE, LEE, KANG, 2005). Nonaka e Takeuchi (1997) apontam que os conhecimentos precisam ser compartilhados na organização para que esta esteja capacitada a sistematizar e criar conhecimentos, ou seja, inovar ao difundi-lo, incorporando-o em produtos, serviços e sistemas (NONAKA; TAKEUCHI, 1997).

As práticas para a distribuição do conhecimento são favorecidas com o uso de ferramentas com o apoio das tecnologias de informação, contudo, a transferência de informações e conhecimentos entre pessoas, entre escalões diferentes e entre diferentes áreas funcionais são desenvolvidas a partir de cada realidade organizacional, que envolve desde questões de poder e motivação até a hierarquia e infraestrutura (SALIM, 2003).

Os estudos sobre compartilhamento de conhecimento são vastos, todavia, na sua grande maioria referem-se às organizações privadas (ALCARÁ et al., 2009; BARBOSA; MONTEIRO; FREITAS, 2012; RIZZON; TAMIOSSO; FACHINELLI, 2016; SORDI, NAKAYAMA; BINOTTO, 2018; FAROOQ, 2018). A literatura é mais tímida 
nos estudos desse tema voltados às organizações públicas (COELHO, 2004; BATISTA, 2006; TONET; PAZ, 2006; FRESNEDA; GOULART, 2007; ANGELIS, 2011; YUSOF et al., 2012; BRITO et al., 2017). Ainda mais escassos são os estudos que abordam o tema relacionado às instituições de ensino (RODRIGUES; MACCARI, 2003; MUSSI; ANGELONI, 2011; TEODOROSKI et al., 2013; CAJUEIRO; SICSÚ; RIBEIRO, 2018; AGUIAR FILHO; NASSIF, 2016) especialmente as públicas (MARTINS, 2007; CARDOSO; MACHADO, 2008). Destes últimos, uma grande parcela aborda o compartilhamento do conhecimento científico ou acadêmico (LEITE; COSTA, 2006; SCHENKEL, 2008; NEELY; COGGBURN, 2017) e não o gerenciamento do conhecimento no âmbito organizacional.

As instituições públicas de ensino trabalham diretamente com o compartilhamento do conhecimento, visto que criam e transferem conhecimentos para a sociedade. Elas apresentam características típicas das organizações públicas que as diferenciam das privadas, como por exemplo, o caráter eletivo e temporário das equipes gestoras, a rotatividade de servidores entre setores, a escassez de recursos e a estabilidade da carreira pública. Além disso, é preciso considerar a especificidade de uma instituição de ensino que é a sua atividade-fim, ou seja, o ensino, a pesquisa e a extensão. Essas peculiaridades reforçam a importância da análise da eficácia das ferramentas adotadas por uma instituição pública de ensino para efetivar o compartilhamento do conhecimento organizacional. Assim, este estudo objetiva identificar e analisar as ferramentas de compartilhamento do conhecimento utilizadas pelo Instituto Federal de Santa Catarina, no âmbito organizacional.

\section{GESTÃO DO CONHECIMENTO}

O aspecto central da sociedade do século XXI é a caracterização do conhecimento como o ativo de produção mais importante, estando à frente dos ativos tradicionais como mão de obra, capital e tecnologia. $O$ conhecimento é, também, o grande diferencial competitivo das empresas e dos países. Consolida-se como a chave para o êxito organizacional (ZABOT; SILVA, 2002; DRUCKER, 1993; HERRERA, 2008; LAKSHMAN, 2009).

A literatura é ampla na categorização do conhecimento. Muitos autores propõem a sua divisão em dois tipos principais: conhecimento tácito e conhecimento explícito. Nonaka e Takeuhi (1997), desenvolvedores da Teoria de Criação do Conhecimento, definem que o conhecimento tácito é da ordem do indivíduo, suas particularidades, suas vivências e suas experiências que colaboram para o crescimento pessoal e grupal, sendo difícil de formalizar, o que dificulta a transmissão e compartilhamento com outros. Já o conhecimento explícito é da ordem da expressão, possível de ser quantificado, sendo expresso em informações. É facilmente comunicado e compartilhado sob a forma de dados brutos, fórmulas científicas, procedimentos codificados ou princípios universais (NONAKA; TAKEUHI, 1997).

A GC tem destaque como elemento estratégico no ambiente corporativo e como tema de discussões acadêmicas. Nos últimos anos, diversos autores discutiram a GC nas organizações. A quantidade de autores que estudam o tema se reflete na diversidade de conceitos para o termo GC, conforme apresentados no Quadro 1. O Quadro não abrange todos os conceitos existentes na literatura. No entanto, fornece um entendimento abrangente sobre como são tratados os conceitos do termo GC.

Quadro 1 - Conceitos de gestão do conhecimento

\begin{tabular}{|l|c|}
\hline \multicolumn{1}{|c|}{ Conceito } & \multicolumn{1}{|c|}{ Autor } \\
\hline $\begin{array}{l}\text { A capacidade de uma organização de criar conhecimento, disseminá-lo } \\
\text { na organização e incorporá-lo em seus produtos, serviços e sistemas. }\end{array}$ & Nonaka e Takeuchi (1997) \\
\hline $\begin{array}{l}\text { A gestão do conhecimento é um processo de coleta, distribuição e } \\
\text { utilização eficiente dos recursos de conhecimento em toda a } \\
\text { organização. }\end{array}$ & Davenport e Prusak (1998) \\
\hline $\begin{array}{l}\text { Um processo de criação, validação, apresentação, distribuição e } \\
\text { aplicação que permite a uma organização aprender, refletir, } \\
\text { desaprender e reaprender, sendo estas cinco fases consideradas } \\
\text { essenciais para construção, manutenção e reabastecimento das } \\
\text { competências principais. }\end{array}$ & Bhatt (2001) \\
\hline
\end{tabular}


Gerenciamento de processos que governam a criação, disseminação e utilização do conhecimento por meio da fusão de tecnologias, estruturas organizacionais e pessoas para criar um aprendizado mais efetivo, resolver problemas e tomar decisão em uma organização.

Processo que facilita a partilha de conhecimentos e estabelece aprendizagem como um processo contínuo dentro de uma organização. Planejar, dirigir e controlar os fluxos de conhecimento que são produzidos na organização, bem como ações desenvolvidas para obter, aproveitar e melhorar o conhecimento que a organização necessita para ser competitiva.

Um conjunto global de processos que são postos em prática com a finalidade de identificação das fontes de dados e informações relevantes nas organizações e eventual conversão desses dados e informações em

Na Ubon e Kimble (2002) conhecimento para sua posterior divulgação em pontos diferentes da organização onde eles são necessários.

Fonte: Elaborado pelos autores (2018).

A partir dos conceitos apresentados no Quadro 1, identifica-se que a GC está relacionada com os padrões de interação entre as pessoas, as tecnologias e os processos organizacionais na criação e utilização do conhecimento para resolução dos problemas organizacionais, bem como para a tomada de decisão. Cabe salientar que, o conhecimento organizacional não é apenas resultante da soma do conhecimento individual. O conhecimento da organização é formado por meio de padrões únicos de interações, as quais não são facilmente imitadas por outras organizações, uma vez que são moldadas pela cultura e história de cada organização (BHATT, 2001).

A interação de pessoas, tecnologias e processos são os elementos viabilizadores da GC que acontece em etapas de forma cíclica, conforme Figura 1 (GOZZI, 2012).

Figura 1 - Ciclo da Gestão do Conhecimento

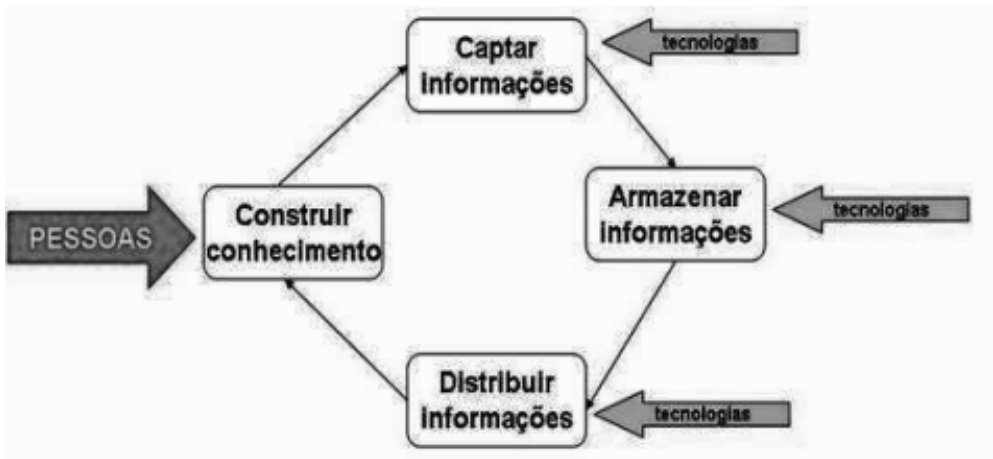

Fonte: Gozzi (2012, p. 10).

As soluções tecnológicas permitem a captação, o armazenamento e a distribuição das informações. A organização e o acesso às informações são necessários para a construção do conhecimento. Contudo, é um equívoco compreender a GC como gestão de tecnologias. A tecnologia se apresenta como importante, mas não é a solução, pois é necessário que a tecnologia usada nas organizações esteja alinhada com as pessoas e processos organizacionais, caso contrário, ficará inutilizada (SERVIN; DE BRUN, 2005).

A construção do conhecimento compete às pessoas. Assim, a GC tem como pressuposto que o recurso mais valioso das organizações reside nas pessoas, pois depende delas criar conhecimento, compartilhá-lo com a organização e usá-lo para melhorar o resultado organizacional (SERVIN; DE BRUN, 2005; BHATT, 2001). Portanto, uma cultura voltada para a GC é necessária e um grande desafio. De modo geral, para criar um 
ambiente de conhecimento, normalmente, são requeridas mudanças na cultura organizacional, mudando o comportamento e os padrões de trabalho das pessoas na perspectiva de longo prazo (SERVIN; DE BRUN, 2005).

A implantação da GC em uma organização direciona os esforços para a aplicação do conhecimento da força de trabalho para alcançar objetivos organizacionais específicos. Isso demanda que as pessoas tenham o conhecimento disponível onde e quando precisarem - o conhecimento certo, no lugar certo, no tempo certo. Para tanto, além da tecnologia, a GC se preocupa com a facilitação de processos pelos quais o conhecimento é criado, compartilhado e usado nas organizações (SERVIN; DE BRUN, 2005), ou seja, os processos para a GC a partir de modelos da GC.

\subsection{Modelos da Gestão do Conhecimento em instituições públicas}

Na literatura são identificadas diversas propostas para diagnosticar e mensurar práticas da GC. Citase, como exemplo, Nonaka e Takeuchi (1997) com a espiral do conhecimento organizacional; Davenport e Prusak (1998) com as três fases da GC; Teixeira Filho (2001) com os pilares da GC; Terra (2005) com o modelo das sete dimensões; Cavalcanti (2001) com o modelo dos quatro capitais, Probst, Raub e Romhardt (2002) com os elementos construtivos; Angeloni (2002) por meio das três dimensões da GC; Choo (2003) com as arenas do conhecimento, dentre outros.

Cada modelo da GC apresenta suas características e fases, contudo, o estudo de Miranda (2004) identificou uma similaridade nas fases em dez modelos analisados. O referido autor observou que as fases de criação, aquisição, armazenamento, compartilhamento, transferência e aplicação são as mais frequentes nos modelos. Em síntese, a GC permite: a) criar novos conhecimentos, inovando a partir de novos produtos, processos e serviços; b) adquirir conhecimentos, por meio de suas diversas fontes; c) armazenar conhecimentos, retendo o conhecimento em seus processos, pessoas e bases; $d$ ) compartilhar conhecimentos, promovendo a interação social; e) usar conhecimento, aplicando o conhecimento em novos contextos (SCHONS, 2008) em diversos tipos organizacionais.

No entanto, alguns autores defendem um modelo de GC específico para o setor público (CONG, PANDTA, 2003; ABDULLAH, DATE, 2009) em vez de adotar os modelos desenvolvidos com base no setor privado ou universais, tendo em vista algumas de suas características, o seu perfil e seus objetivos. Nas instituições públicas, a GC está associada à eficiência, eficácia, efetividade social, ao desenvolvimento econômico e aos princípios da legalidade, impessoalidade, publicidade, moralidade e eficiência, questões específicas deste tipo organizacional (BATISTA, 2012). Contudo, na literatura ainda há poucos estudos sobre modelos de GC para instituições públicas (BATISTA, 2012).

O estudo de Batista (2012) desenvolveu uma proposição de modelo de GC para organizações públicas que considera a: a) dimensão do cidadão-usuário e a sociedade, que são os públicos-alvos destas instituições e a b) dimensão dos resultados baseados no conceito de inovação, que no setor público significa aumentar a eficiência e melhorar a qualidade dos serviços públicos, conforme pode ser visualizado na Figura 2. 


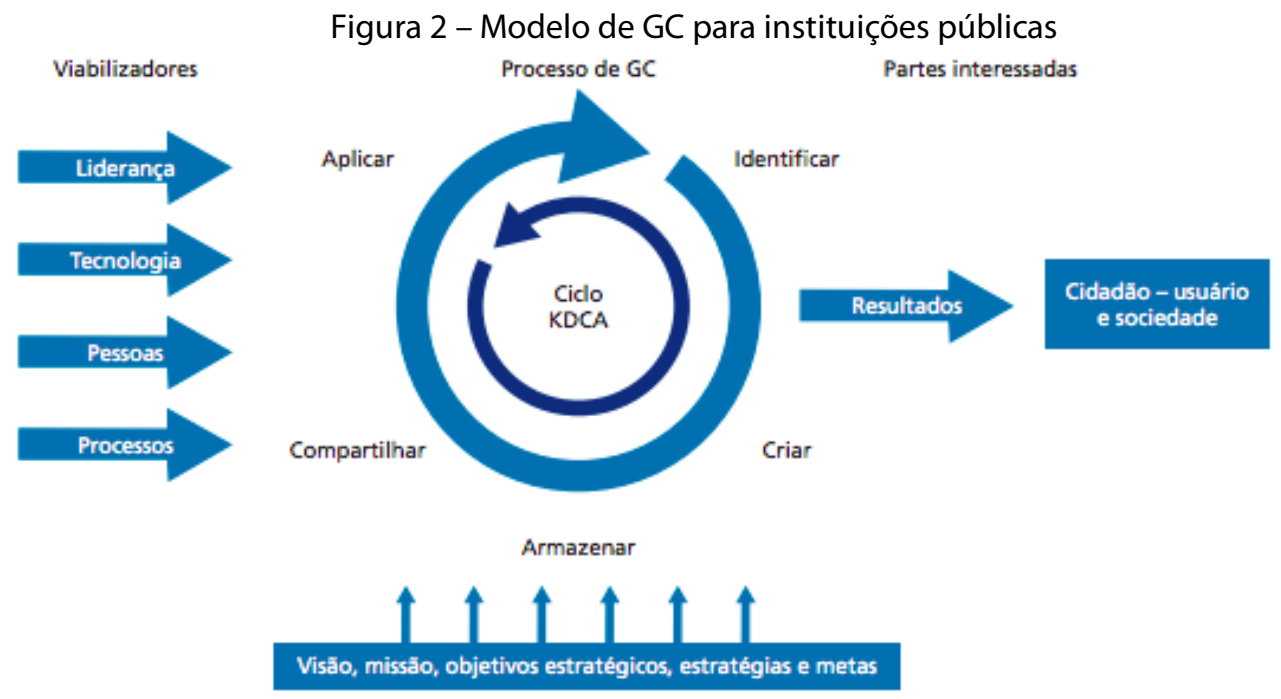

Fonte: Batista (2012).

Em relação às abordagens clássicas de modelo de GC, identifica-se a inclusão da liderança como elemento-chave para a implantação da GC no setor público (BATISTA, 2012; LIN, 2007; WANG; NOE, 2010).

De acordo com Batista (2012), a liderança tem um papel fundamental no processo, pois compete a ela reforçar a visão, objetivos e estratégias da GC; estabelecer a estrutura de governança para formalizar os projetos de GC; alocar recursos financeiros para viabilizar os projetos de GC e definir a política de proteção do conhecimento.

As pessoas também se configuram como elementos-chave na implantação da GC nas instituições públicas. Por isso, a questão de uma cultura organizacional que favoreça a implantação da GC também precisa ser discutida. A pesquisa de Batista (2006) reforça o papel das pessoas na GC, destacando sua contribuição no processo do trabalho e na cultura organizacional.

Já quanto aos processos de GC, Batista (2012) idealiza um conjunto de etapas para atender as especificidades das instituições públicas com 5 etapas: identificação, criação, armazenamento, compartilhamento e aplicação. Contudo, quando é comparado com o modelo de Miranda (2004)1, identificase uma similaridade quanto às etapas dos processos de GC, conforme o comparativo apresentado no Quadro 2.

Quadro 2 - Comparativo entre Processos de GC

\begin{tabular}{|c|c|}
\hline $\begin{array}{c}\text { Processos de GC } \\
\text { (Batista, 2012) }\end{array}$ & $\begin{array}{c}\text { Processos de GC } \\
\text { (Miranda, 2004) }\end{array}$ \\
\hline Identificação & Criação \\
\hline Criação & Aquisição \\
\hline Armazenamento & Armazenamento \\
\hline Compartilhamento & Compartilhamento \\
\hline Aplicação & Aplicação \\
\hline
\end{tabular}

Fonte: elaborado pelos autores (2018) a partir de Batista (2012) e Miranda (2004).

1 Miranda (2004) em sua tese propôs um modelo de gestão do conhecimento de forma sistêmica, considerando o conhecimento como elemento estratégico e atendendo diversos tipos organizacionais. 
Dentre as fases identificadas no processo de GC, reconhece-se a importância de cada uma para que o conhecimento seja utilizado de forma eficiente e eficaz pelas organizações. Contudo, este estudo tem seu foco em uma etapa específica desse processo que é o compartilhamento do conhecimento em instituições públicas. Neste tipo organizacional, o compartilhamento do conhecimento exige planejamento, estabelecendo a GC alinhada à sua missão, visão e estratégias e considerando os aspectos da burocracia dos processos e a hierarquia organizacional (BATISTA, 2006).

\subsection{0 compartilhamento do conhecimento em instituições públicas}

O processo de compartilhamento, também chamado de distribuição e transferência (DAVENPORT, PRUSAK, 1998; PROBST, RAUB, ROMHARDT, 2002), compreende o processo de comunicação do conhecimento, o qual permite o seu entendimento e uso por parte do destinatário. Ou ainda, a transferência de conhecimentos tácitos e explícitos por meio de práticas formais e informais (GROTTO, 2002).

Para o compartilhamento ocorrer, as pessoas precisam estar voltadas para a disseminação do conhecimento, compartilhando suas experiências e ideias. Segundo Davenport e Prusak (1998), a organização só se beneficia como um todo quando o conhecimento é difundido e transferido, ou seja, compartilhado. Neste processo, há duas estratégias utilizadas: a transferência espontânea e não estruturada, que está ligada aos conhecimentos tácitos, e a transferência estruturada, que se refere ao conhecimento explícito.

A transferência espontânea e não estruturada, comumente tácita, abrange as comunicações informais, workshops, reuniões face a face, trabalho em conjunto, jantares em grupo, salas de bate papo, entre outros (DAVENPORT; PRUSAK, 1998). Em resumo, não são utilizadas ferramentas tecnológicas. O brainstorming, que consiste em técnica que objetiva o surgimento de ideias sem o julgamento prévio das mesmas, também pode ser considerado uma ferramenta dessa categoria.

A transferência estruturada refere-se aos processos de transferência de conhecimento explícito por meio de mídias físicas (hardwares, equipamentos, manuais, documentos físicos etc.) e/ou digitais (softwares, aplicativos, documentos virtuais etc.), ou seja, com o apoio da tecnologia. Com um estudo de softwares, Carvalho (2000) elenca a intranet, mapas de conhecimento, infomapping, gestão eletrônica de documentos (GED) e groupware como ferramentas tecnológicas de apoio à GC. Destaque-se, ainda, a videoconferência, a internete os sistemas especialistas (DAVENPORT; PRUSAK, 1998).

Um estudo realizado, na E-Consulting Corp (2004), mapeou de forma quantitativa o uso de algumas ferramentas no compartilhamento do conhecimento e constatou que o e-mail é utilizado por $84,2 \%$ dos entrevistados, internet por $64,2 \%$, debates por $46,3 \%$, listas de discussões por $29,0 \%$, chats programados por $22,5 \%$, mensagens instantâneas por $18,8 \%$ e multiplicadores de conhecimento por 16,4\%. Em resumo, identificou-se na literatura, ferramentas tecnológicas e não tecnológicas que podem apoiar o compartilhamento do conhecimento, conforme o Quadro 3.

Quadro 3 - Ferramentas de apoio ao compartilhamento do conhecimento

\begin{tabular}{|c|c|}
\hline Ferramentas tecnológicas & Ferramentas não tecnológicas \\
\hline E-mail & Debates \\
\hline Internet & Multiplicadores de conhecimento \\
\hline Intranet & Comunicação informal \\
\hline Mapas de conhecimento & Workshop \\
\hline Gestão eletrônica de documentos (GED) & Reuniões face a face \\
\hline Groupware & Brainstorming \\
\hline Chats programados (webe videoconferência) & Simulações \\
\hline Sistemas especialistas & Manuais \\
\hline Listas de discussão & \\
\hline
\end{tabular}




\begin{tabular}{|c|l|}
\hline Mensagens instantâneas & \\
\hline Infomapping & \\
\hline
\end{tabular}

Fonte: elaborado pelos autores (2018).

Como já exposto, a literatura apresenta poucos estudos específicos sobre a GC em instituições públicas. Batista (2012) apresenta um estudo sobre as ferramentas e técnicas mais usuais para a disseminação do conhecimento no setor público, conforme o Quadro 4.

Quadro 4 - Ferramentas de compartilhamento do conhecimento em instituições públicas

\begin{tabular}{|c|}
\hline Melhores práticas \\
\hline Memória organizacional/ lições aprendidas \\
\hline Banco de conhecimento \\
\hline Sistema de inteligência organizacional \\
\hline Mapeamento e auditoria do conhecimento \\
\hline Sistema de gestão por competência \\
\hline Banco de competências organizacionais \\
\hline Banco de competências individuais \\
\hline Gestão do capital intelectual \\
\hline Fonte Batista (2012).
\end{tabular}

Fonte: Batista (2012).

Além da importância das ferramentas, cabe ressaltar que o compartilhamento de conhecimento é um processo que sofre influência da cultura organizacional. A capacidade de uma organização difundir práticas inovadoras depende, entre outros fatores, de uma cultura organizacional que encoraje a reciprocidade, a curiosidade e a reflexão, além das fronteiras internas e externas e de infraestruturas de aprendizagem (SENGE, 1999).

Com o embasamento teórico sobre GC, seus modelos e ferramentas, observando as especificidades das instituições públicas, a próxima seção apresenta o caminho percorrido para a análise das ferramentas utilizadas pelo Instituto Federal de Santa Catarina para o compartilhamento do conhecimento.

\section{PROCEDIMENTOS METODOLÓGICOS}

Os procedimentos metodológicos se referem ao caminho que foi adotado para alcançar os objetivos traçados, ou seja, descreve-se a forma de pensar, agir e compreender a realidade (MINAYO et al., 1994; TAYLOR; BOGDAN, 1987). A partir de uma perspectiva objetiva da realidade, a investigação proposta seguiu as orientações da pesquisa qualitativa (YIN, 1994).

A instituição selecionada foi o Instituto Federal de Santa Catarina (IFSC) por demonstrar preocupação com os processos de GC, denotada por sua previsão e importância percebida no Plano de Desenvolvimento Institucional (PDI) 2015-2019, que prevê a fomentação do desenvolvimento da cultura de compartilhamento de conhecimentos, de informações e de práticas de gestão do conhecimento e inovação no IFSC (IFSC, 2018). 
Foram coletados dados primários e secundários a partir das técnicas de análise documental (BARDIN, 1979) e entrevista semiestruturada (LIMA, ALMEIDA e LIMA, 1999). Os documentos institucionais analisados foram o PDI 2015 - 2019, a Política de Comunicação do IFSC, a Resolução número 45, de 18 de dezembro de 2014, que aprova a Política de Formação do IFSC, a Resolução CONSUP número 52, de 24 de outubro de 2016, que institui a Política de Segurança da Informação no IFSC, entre outros documentos disponibilizados no site institucional. A entrevista foi realizada pessoalmente, em evento único, e com duração de aproximadamente uma hora e trinta minutos, com um gestor da Diretoria de Gestão do Conhecimento (DGC), denominado de "Entrevistado", mediante a sua disponibilidade. A entrevista foi gravada para facilitar a análise dos dados coletados. A DGC foi selecionada pelo fato de ter a competência de fomentar o desenvolvimento da cultura de compartilhamento de conhecimentos, de informações e de práticas de GC e inovação no IFSC (IFSC, 2018).

A análise dos dados que objetiva organizar e sumarizar os dados para o fornecimento de respostas para o objetivo da investigação, foi desenvolvida pela análise de conteúdo, que consiste em um conjunto de técnicas de análise das comunicações, que utiliza procedimentos sistemáticos e objetivos de descrição do conteúdo das mensagens (BARDIN, 1979).

\section{ESTUDO DE CASO}

O IFSC foi criado em 1909 por meio do decreto no 7.566, de 23 de setembro, pelo presidente Nilo Peçanha, como Escola de Aprendizes Artífices de Santa Catarina. Em 1937, passou a se chamar Liceu Industrial de Florianópolis e cinco anos mais tarde transformou-se em Escola Industrial de Florianópolis. Em 1965 passou a se chamar Escola Industrial Federal de Santa Catarina e, três anos depois, a Instituição tornou-se Escola Técnica Federal de Santa Catarina (ETF-SC). Em 2002, foi transformada oficialmente em Cefet-SC e, por fim, em 2008, transformou em Instituto Federal de Educação, Ciência e Tecnologia de Santa Catarina (IFSC, 2018).

\subsection{A gestão e o compartilhamento do conhecimento no IFSC}

Entre 2009 e 2015, o IFSC passou por uma forte etapa de expansão, com a implantação de quinze campiespalhados pelas cinco regiões do Estado, totalizando, atualmente, vinte e dois campus, que atuam nas ofertas de formação básica, técnica e tecnológica, cursos de qualificação profissional, técnicos, de graduação e pós-graduação (IFSC, 2018).

O IFSC é caracterizado por uma estrutura conjugada sob a forma de rede, que permite a integração e o padrão nas ações de planejamento e execução. A Instituição tem uma estrutura administrativa composta por: a) Gabinete da Reitoria; b) Pró-Reitoria de Administração; c) Pró-Reitoria de Desenvolvimento Institucional; d) Pró-Reitoria de Ensino; e) Pró-Reitoria de Extensão e Relações Externas; f) Pró-Reitoria de Pesquisa, PósGraduação e Inovação; 22 campus em todo território de Santa Catarina (IFSC, 2018).

Para auxiliar no alcance de seus objetivos institucionais, o IFSC conta com uma diretoria que cuida dos processos de GC, a DGC, que é vinculada a Pró-Reitoria de Desenvolvimento Institucional (IFSC, 2010; IFSC, 2018). A DGC tem papel importante no compartilhamento de conhecimento, visto que tem a competência de "fomentar o desenvolvimento da cultura de compartilhamento de conhecimentos, de informações e de práticas de gestão do conhecimento e inovação no IFSC" (IFSC, 2018, p. 5). E, ainda, é responsável por: a) estabelecer diretrizes e coordenar a elaboração do planejamento institucional, em consonância com o PDI e o Projeto Pedagógico Institucional; b) coordenar o processo de mapeamento e documentação dos processos institucionais; c) coordenar a elaboração do planejamento anual das atividades de gestão do conhecimento e d) coordenar a elaboração dos Manuais de Gestão do IFSC (IFSC, 2010).

Cabe à DGC, por meio da Coordenadoria de Processos e Normas, o mapeamento e modelamento dos processos institucionais, compartilhando com os servidores o conhecimento sobre os processos do IFSC, em 
busca da melhoria contínua, da padronização dos processos internos, eliminando desperdícios e atividades que não geram resultados positivos para a sociedade (IFSC, 2017), visando atender ao GESPUBLICA².

A estrutura, políticas e alinhamento estratégico do IFSC quanto ao estímulo e promoção da GC expressos em seus documentos (PDI, PPI, Regimento Interno), realizado por meio de uma diretoria de GC vai ao encontro com o que abordam os autores da área para a construção de uma cultura organizacional em prol do conhecimento. Davenport e Prusak (1998), apontam que um gestor designado para cuidar das atividades do conhecimento envolve habilidades relacionadas à implementação de uma cultura organizacional de aprendizado, ao desenvolvimento da infraestrutura facilitadora e à competência para tornar a atividade do conhecimento economicamente compensadora.

Indo na direção da compreensão do compartilhamento do conhecimento no IFSC, passamos por Tonet e Paz (2006) que afirmam que o processo de compartilhamento de conhecimento deve considerar quatro elementos semelhantes aos que sustentam o processo de comunicação entre pessoas: a fonte do conhecimento a ser compartilhado, o destinatário desse conhecimento, o conhecimento em si e o contexto em que o compartilhamento de conhecimento ocorre. Assim, observando o IFSC, além da identificação da DGC como uma estrutura orientadora sobre os processos da GC, encontramos a Política de Comunicação Institucional ${ }^{3}$ que traz indícios de como a comunicação está relacionada com o compartilhamento do conhecimento.

A comunicação interna no IFSC compreende os processos, ações, estratégias, veículos ou canais e manuais que se destinam ao relacionamento entre o instituto e seus públicos internos e externos. Está associada ao processo de gestão e à cultura organizacional e não se limita aos veículos formais de comunicação, como: intranet, informativos do campus e mídias sociais. A Política de Comunicação do IFSC incorpora os contatos interpessoais, as relações entre servidores e todas as instâncias que permitem a interação dos (e com) os públicos internos, visando o desenvolvimento pessoal e profissional (IFSC, 2014). Ainda, estimula a participação e o feedback como instrumentos para a construção de uma gestão democrática e dialógica.

Outro elemento de destaque para o compartilhamento do conhecimento diz respeito ao desenvolvimento e aprendizado, ou melhor, a gestão do aprendizado (TERRA, 2005). O IFSC tem instituída a Política de Formação do IFSC ${ }^{4}$, que constitui um conjunto de ações distribuídas em sete programas voltadas para o desenvolvimento dos seus servidores e demais profissionais de instituições de ensino e ampliação das oportunidades de acesso a diferentes possibilidades de cursos de formação: qualificação, capacitação e treinamento, por meio de ações de ensino, pesquisa e extensão (IFSC, 2014).

Atualmente, a Política de Formação é gerida por um comitê e a implementação de suas ações fica sob a responsabilidade do Centro de Referência em Formação e EaD, uma diretoria da Pró-Reitoria de Ensino. Outro setor que tem relação direta com a formação dos servidores é a Coordenadoria de Capacitação e Avaliação, vinculada à Diretoria de Gestão de Pessoas (DGP) que, por sua vez, está subordinada à Diretoria Executiva. Esse setor é responsável pela elaboração do Plano Anual de Capacitação $(\mathrm{PAC})^{5}$ e pela coordenação do Programa de Avaliação de Desempenho dos servidores, que visa diagnosticar as carências e subsidiar o desenvolvimento de programas de capacitação.

2 A gestão de processos no setor público brasileiro foi inserida como parte de um conjunto de métodos e soluções do Programa Nacional de Gestão Pública e Desburocratização (GESPÚBLICA), instituído pelo Decreto 5.378, de 23 de fevereiro de 2005, que vem ao encontro da criação de um modelo de excelência em gestão focado em resultados e orientado para o cidadão.

3 A Política de Comunicação do IFSC foi instituída pela Resolução no 26/2013/CONSUP apresenta o conjunto de princípios, diretrizes e estratégias que objetivam orientar o relacionamento do IFSC com os seus públicos estratégicos com o pressuposto de vincular o processo de gestão e a cultura organizacional do instituto.

$4 \quad$ A Política de Formação do IFSC foi criada pela Resolução no 45, de 18 de dezembro de 2014.

O Plano de Anual de Capacitação (PAC) é configurado como norteador das ações de aprendizagem do processo de capacitação, definindo diretrizes gerais e metodologias voltadas para o desenvolvimento de competências dos servidores do IFSC, atendendo a Portaria $\mathrm{n}^{\mathrm{o}} 208$ de 25 de julho de 2006 (Ministério do Planejamento, Orçamento e Gestão) e o Decreto n ${ }^{\circ}$ 5.707, de 23 de fevereiro de 2006. 
Identifica-se, portanto, uma estrutura organizacional voltada para o desenvolvimento e formação, por meio da gestão de capacitação por competências ${ }^{6}$, em diferentes órgãos. Esse fato pode ser interessante para a disseminação de uma cultura de aprendizagem de forma institucional, desde que seja de forma articulada e ordenada entre os setores envolvidos, pois os documentos não detalham como acontece na prática e corre-se o risco de ações isoladas e desconectadas.

Após compreender a estrutura, política e cultura do compartilhamento do conhecimento no IFSC, a próxima seção apresenta as práticas de disseminação por meio das ferramentas utilizadas na Instituição.

\subsection{Ferramentas de compartilhamento de conhecimento no IFSC}

A gestão eficiente do conhecimento requer um conjunto de ferramentas para permitir a ampliação do seu alcance e a aceleração da velocidade de sua disseminação (UBON, KIMBLE, 2002). No IFSC, as principais ferramentas utilizadas no processo de compartilhamento de conhecimento na Instituição são, de acordo com o entrevistado: o e-mail institucional, as reuniões presenciais ou via vídeo/web conferência, as conversas face a face, uso do disco virtual institucional e do google drive, os fóruns de discussão, o uso do Ambiente Virtual de ensino e Aprendizagem (AVA), o mapeamento de competências, as capacitações, o mapeamento de processos, a intranet, o site institucional, o Sistema Integrado de Gestão (SIG), o contato telefônico, os aplicativos de mensagens instantâneas, a internet, os grupos de trabalho (GTs) e os manuais e tutoriais.

Dentre as ferramentas utilizadas, o entrevistado cita como as de maior relevância, por sua perceptível efetividade e aceitação por parte dos usuários, as reuniões presenciais e o uso do e-mail institucional. As reuniões presenciais e as conversas face a face são, culturalmente, os principais meios não tecnológicos utilizados, ao passo que o uso do e-mailinstitucional se destaca como o principal meio tecnológico utilizado para efetivar o compartilhamento de conhecimento dentro da Instituição. Para o entrevistado, o uso da comunicação via e-mailinstitucional é consolidado como o carro-chefe do compartilhamento de informações e conhecimentos na rede, principalmente, pelo caráter oficial deste instrumento e pelo alto grau de aceitação do mesmo pelos usuários.

Todavia, na busca de informações e esclarecimento de dúvidas mais urgentes o uso de aplicativos de mensagens instantâneas é predominante na Instituição, mesmo não sendo uma ferramenta institucional, conforme descreve o entrevistado "hoje o whatsapp ele é dinâmico, é rápido [...] e ajuda muito na prática. Aí tem a questão de não ser uma ferramenta institucional, formal". De acordo com ele, as ponderações e discussões acerca do uso desses aplicativos na Instituição está na sua utilização que, muitas vezes, ultrapassa as barreiras do "horário de trabalho" e da privacidade.

A troca e a combinação de conhecimento pelo uso de aplicativos de mensagens instantâneas, somado ao contato via telefone são citados pelo entrevistado como os principais meios informais de compartilhamento de conhecimento entre campi e entre campus/Reitoria, principalmente, devido às suas distâncias geográficas. Para ela, a facilidade e agilidade de transmissão e recebimento de informações por meio dessas ferramentas é o que incita essa ampla utilização.

Para armazenar, gerenciar e efetivar o compartilhamento de documentos e informações em rede, a Instituição utiliza diferentes plataformas. Todavia, esse modo de disponibilização multifacetada pode ser um entrave à celeridade e à eficiência dos processos, visto que em alguns casos não existe uma comunicação eficiente para que os servidores saibam "onde encontrar o que". De acordo com o entrevistado, "a questão de tu ter arquivos em vários locais, isso, na organização administrativa, é bastante complicado."

O site institucional é uma das plataformas utilizadas para a disponibilização de documentos e informações, porém é mais focado no público externo. Já a intranet institucional é uma plataforma utilizada somente pelo público interno. Para o entrevistado, a importância da intranet institucional pode ser

6 O Decreto $\mathrm{n}^{\mathrm{0}} 5.707$, de 23 de fevereiro de 2006 instituiu a gestão da capacitação por competências como referência para a gestão de pessoas do setor público. O IFSC, a partir de 2018, iniciou o mapeamento de competências como ação estratégica de gestão e para a promoção do desenvolvimento dos servidores da Instituição. 
considerada mediana quanto à sua relevância de uso e eficácia, visto que esta ferramenta é utilizada, essencialmente, para disponibilizar manuais, tutoriais, modelos de documentos e processos e editais internos.

Outra plataforma disponível é o disco virtual institucional, que é um serviço de armazenamento de arquivos na nuvem exclusivo para conta dos servidores do IFSC, sendo possível acessar os arquivos armazenados no serviço a partir de qualquer computador ou dispositivos móveis (IFSC, 2018). Apesar de ser uma tecnologia que contribui para o compartilhamento de arquivos e documentos não é utilizado tanto como o esperado. Em geral, a ferramenta é normalmente substituída pelo uso do google drive, um software de armazenamento e sincronização de arquivos gratuito que tem mais funcionalidades. Uma deficiência apontada pelo entrevistado no uso do disco virtual institucional é a de que este não comporta, a edição de um documento por múltiplas pessoas simultaneamente, uma prática bastante utilizada pelos gestores da Instituição, que disponibilizam documentos a serem aprovados em fóruns e colegiados para que todos os participantes acessem, analisem e façam contribuições.

O Sistema Integrado Gerencial (SIG) é o principal sistema que o IFSC utiliza para organizar as informações e os processos institucionais. O SIG divide-se por módulos que abrangem as diferentes áreas de gestão com suas características específicas. $O$ entrevistado aponta que, para isso, é preciso ter as atribuições bastante claras e definidas, o que justifica a necessidade de mapear os processos de todas as áreas institucionais.

Nesse panorama, o entrevistado aponta que o mapeamento dos processos na Instituição ainda está em fase de consolidação, com a atuação da Coordenadoria de Processos e Normas (CPN) que vêm mapeando os processos em todos os setores e departamentos da Instituição, objetivando a difusão do conhecimento por meio de processos padronizados e que garantam maior agilidade, facilitando assim, as transições dos servidores a frente desses processos.

Outro processo de mapeamento existente no IFSC, o mapeamento de competências, somado às entrevistas de devolução da avaliação de desempenho, embasam as decisões sobre necessidades de capacitação na Instituição, elencadas no Plano Anual de Capacitação (PAC) (IFSC, 2018). Para o entrevistado, "um ponto desfavorável é que nem sempre as capacitações atendem no tempo necessário os servidores que necessitam serem treinados, como por exemplo, quando uma nova gestão eleita é empossada ou quando ocorre a remoção de um servidor."

Uma ferramenta utilizada no IFSC que contribui para o mapeamento de competências é o sistema Stela Experta. A plataforma é usada como fonte de informação para geração de relatórios que subsidiam a tomada de decisões estratégicas e a gestão interna do Instituto. Os coordenadores de pós-graduação utilizam a solução como insumo para direcionar de forma mais efetiva editais de seleção e captação de recursos no ambiente educacional. Além disso, por meio dos dados de produtividade dos professores e de seus projetos de pesquisa é possível fazer uma distribuição de disciplinas a serem ministradas e projetos a serem contemplados com laboratórios e aportes financeiros (IFSC, 2018).

Na visão do entrevistado, a Instituição não tem uma política de compartilhamento de conhecimento ou capacitação orientada para o processo de transição de equipes gestoras ou para remoções de servidores. Entretanto, ele aponta que isso já é visto como uma necessidade pela alta gestão, citando seu próprio caso como exemplo, que ao assumir a DGC não teve um repasse de conhecimentos mínimos pertinentes à função que passaria a exercer, sobre o qual discorreu: "quando isso ocorre, o servidor acaba tendo que recorrer às áreas na Reitoria [...]. Mas essa falha, na maioria das vezes, não é culpa do servidor, mas sim uma questão cultural, uma deficiência da Instituição". No entanto, apesar de não institucionalizado, o entrevistado discorre que "muitos campus adotam a prática de realizar um período de transição entre servidores, principalmente, nos casos de remoção entre campus, situação em que o servidor só é liberado para assumir a vaga na outra unidade após a chegada e a capacitação do seu substituto".

Atualmente, a Instituição tem adotado a prática de realizar capacitações e, até mesmo, reuniões utilizando ferramentas de teletransmissão, como a videoconferência e a web conferência, assim como pelo acesso ao AVA. Nesse sentido, o entrevistado afirma que "não é feito tanto como deveríamos, talvez até por uma questão cultural dos servidores de acharem que se produz mais numa reunião presencial [...], mas é uma ótima ferramenta em questão de custos e agilidade". Ele aponta ainda que o uso da internet auxilia e facilita o 
processo de compartilhamento, como a prática institucional de transmitir ao vivo, pela IFSC TV (canal do IFSC no youtube), as reuniões do Colégio de Dirigentes (CODIR) e do Conselho Superior (CONSUP), instâncias máximas do IFSC.

As reuniões presenciais, por sua vez, têm grande aceitação e ocorrem com bastante frequência na Instituição, assim como as conversas face a face, considerada pelo entrevistado como uma ferramenta prática e rápida para se tirar dúvidas e obter conhecimentos.

Uma prática comum na Instituição é o compartilhamento de boas práticas entre áreas, entre campie entre campus/Reitoria. $\mathrm{O}$ entrevistado pondera que: "[...] alguns processos não possuem padronização em nível de rede, então cada campus faz de uma forma, porém a partir do momento que um fica sabendo que alguém fez de um jeito que deu certo, naturalmente, adota a prática". O compartilhamento do conhecimento ocorre, ainda, por intermédio de trabalho entre grupos, que se organizam em forma de Grupos de Trabalho (GTs), comissões, fóruns de discussão por áreas, de forma institucional e grupos de mensagens instantâneas, de forma não oficial.

Identifica-se, ainda, alguns incentivos à inovação e ao compartilhamento de ideias no IFSC, como o "Desafio IFSC de Ideias Inovadoras", que visa premiar práticas inovadoras implementadas, estimulando os servidores a pensarem em novas soluções para melhorar a gestão pública.

As ferramentas para o compartilhamento do conhecimento mapeadas no IFSC, classificadas entre tecnológicas e não tecnológicas são apresentadas no Quadro 6.

Quadro 6 - Ferramentas utilizadas pelo IFSC para compartilhamento do conhecimento

\begin{tabular}{|c|c|}
\hline Ferramentas tecnológicas & Ferramentas não tecnológicas \\
\hline E-mai/Institucional & Reuniões presenciais/Conversas \\
\hline Telefone & Grupos de Trabalho (GTs) \\
\hline Internet & Manuais e tutoriais \\
\hline Web conferência/Videoconferência & Mapeamento de processos \\
\hline Disco virtual/Drive & Capacitação baseada nas competências \\
\hline Intranet & \\
\hline Aplicativo de mensagem instantânea & \\
\hline Sistema Integrado de Gestão - SIG & \\
\hline Fóruns de discussão & \\
\hline Ambiente Virtual de ensino e Aprendizagem & \\
\hline Site Institucional &
\end{tabular}

Fonte: Elaborado pelos autores (2018).

Identifica-se que o IFSC utiliza grande parte das ferramentas levantadas na literatura, incluindo os estudos de Batista (2012) em instituições públicas. Entretanto, algumas ferramentas bastante citadas na literatura não são utilizadas no IFSC ou são utilizadas com pouca frequência, como as sessões de brainstorming e workshop, sendo que esta última foi usada na fase de construção do PDI 2015-2019.

\section{CONSIDERAÇÕES FINAIS}

Nos últimos anos, a GC vem se consolidando como um elemento importante para o desenvolvimento das instituições. A troca de informações e conhecimento é um fator que agrega valor e gera diferencial competitivo para a Instituição. Assim, encontrar meios para facilitar ou promover o compartilhamento de conhecimento é um fator estratégico. 
Neste estudo, buscou-se compreender como ocorre o compartilhamento do conhecimento no Instituto Federal de Santa Catarina. A partir de análise documental e entrevista semiestruturada, levantou-se informações úteis para o desenvolvimento da GC em organizações públicas, mostrando que as pesquisas nessa área podem ser promissoras, visto que os estudos sobre o assunto são ainda incipientes no Brasil. Os principais resultados obtidos indicam que os conceitos sobre compartilhamento do conhecimento podem ser observados na organização estudada. Ressalta-se que os resultados podem ser utilizados para nortear pesquisadores que forem realizar seus estudos no assunto exposto, servindo de insumo para o desenvolvimento de futuras pesquisas, bem como permitir que o descobrimento de novas lacunas compiladas na área.

Além disso, este trabalho contribui para a identificação dos meios tecnológicos e não tecnológicos de compartilhamento do conhecimento de maior relevância utilizados pelo IFSC, como por exemplo, o e-mail institucional e as reuniões presenciais, respectivamente. Os resultados mostraram que existe na Instituição estudada a prática de diversos meios para o compartilhamento do conhecimento, porém é fato que há muito o que evoluir, principalmente no que se refere à integralização das formas adotadas. Entretanto, existe uma conscientização da gestão e um percurso de trabalho neste campo, com programas em andamento, que é a base para a transformação e evolução do compartilhamento na GC. Dessa forma, percebe-se uma oportunidade para produção científica sobre o tema, envolvendo focos diferentes de compartilhamento do conhecimento, como por exemplo, a identificação das ferramentas utilizadas em organizações públicas, bem como a integração dos meios que promovem o compartilhamento do conhecimento.

Por ser um trabalho que se baseia em um estudo de caso, ele apresenta restrições ou cuidados que devem ser lembrados quando utilizado. A escolha de uma única instituição de ensino pública não abrange todas as organizações públicas de ensino, podendo ser necessário estudar outras instituições de ensino governamentais para obter maior completude dos resultados.

Com a predominância de pesquisas sobre o tema, voltado às organizações privadas, identificam-se oportunidades de pesquisa que abordem o compartilhamento do conhecimento, direcionado às organizações públicas.

Assim, sugerem-se novos estudos que analisem como ocorre o compartilhamento do conhecimento em outros tipos de instituições públicas, bem como sejam realizadas futuras pesquisas que comparem os resultados apresentados neste trabalho com os resultados de outros estudos que abordem o compartilhamento do conhecimento em instituições públicas e privadas.

\section{REFERÊNCIAS}

ABDULLAH, T.; DATE, H. Public sector knowledge management: a generic framework. Public Sector Management Review, v. 3, n. 1, p. 1-14, jan./jun. 2009.

ALCARÁ, A. R. et al. Fatores que influenciam o compartilhamento da informação e do conhecimento. Perspectivas em Ciência e Informação, v. 14, n. 1, p. 170-191, 2009.

ANGELIS, C. T. Gestão do conhecimento no setor público: um estudo de caso por meio do método OKA. Revista do Serviço Público, Brasília. v. 62, n. 2, p. 137-166, abr./jun. 2011.

ANGELONI, M. T. Organizações do conhecimento: infra-estrutura, pessoas e tecnologias, São Paulo: Editora Saraiva, 2002.

ARGOTE, L.; MCEVILY, B.; REAGANS, R. Managing knowledge in organizations: an integrative framework and review of emerging themes. Management Science, v. 49, n. 4, p. 571-282, abr. 2003. Disponível em: https://pdfs.semanticscholar.org/aeb2/3992f2a2d386a3a3521908c5354905fc3352.pdf?_ga=2.28317628.105 6227889.1563200359-1874567787.1563200359. Acesso em: 09 abr. 2019.

BARBOSA, J. G. P.; MONTEIRO, L. S. I.; FREITAS, J. A. S. B. Compartilhamento de conhecimento: um estudo de caso em uma instituição financeira. Perspectivas em Gestão \& Conhecimento, João Pessoa, v. 2, n. 1, p. 137-154, 2012. 
BARBOSA. R. R. Gestão da informação e do conhecimento: origens, polêmicas e perspectivas. Informação \& Informação, Londrina, v. 13, n. 1, p. 1-25, 2008.

BARDIN, L. Análise de conteúdo. Lisboa: Edições 70, 1979.

BATISTA, F. F. O governo que aprende: implementando a gestão do conhecimento no executivo federal. In: STAREC, C.; GOMES, E.; BEZERRA, J. (org.) Gestão estratégica da informação e inteligência competitiva. São Paulo: Saraiva, 2006. p. 185-194.

Modelo de gestão do conhecimento para a administração pública brasileira: como implementar a gestão do conhecimento para produzir resultados em benefício do cidadão. Brasília: IPEA, 2012.

BHATT, G. D. Knowledge management in organizations: examining the interaction between technologies, techniques, and people. Journal of Knowledge Management, v. 5, n. 1, p. 68-75. 2001.

BRITO, L. M. P. et al. Programa de mentoria: uma estratégia seminal de compartilhamento do conhecimento em uma empresa pública de energia. RACE: Revista de Administração, Contabilidade e Economia, v. 16, p. 209-234, 2017. Número Especial.

CAJUEIRO, J. L. G.; SICSÚ, A. B.; RIBEIRO, A. R. B. Elementos preliminares para a construção de modelos: a contribuição da gestão do conhecimento para instituições de ensino superior. Revista Gestão Industrial, Ponta Grossa, v. 5, n. 3, p. 219-234, 2018.

CARDOSO, O. N.; MACHADO, R. T. M. Gestão do conhecimento usando data mining: estudo de caso na Universidade Federal de Lavras. Revista de Administração Pública, Rio de Janeiro, v. 42, n. 3, p. 495-528, maio/jun. 2008.

CARVALHO, R. B. de. Aplicações de softwares de gestão do conhecimento: tipologia e usos. Belo Horizonte. 2000. Dissertação (Mestrado em Ciência da Informação) - Programa de Pós-graduação em Ciência da Informação, Universidade Federal de Minas Gerais, Belo Horizonte, 2000.

CAVALCANTI, M. Gestão de empresas na sociedade do conhecimento: um roteiro para a ação. Rio de Janeiro: Campus, 2001.

CHOO, C. W. A Organização do conhecimento: como as organizações usam a informação para criar significado, construir conhecimento e tomar decisões. São Paulo: Editora Senac, 2003.

COELHO, E. M. Gestão do conhecimento como sistema de gestão para o setor público. Revista do Serviço Público, Brasília, v. 55, n 1 e 2, p. 89-115, 2004.

CONG, X.; PANDYA, K. V. Issues of knowledge management in the public sector. Electronic Journal of Knowledge Management, v. 1, n. 2, p. 25-33, 2003.

DAVENPORT, T. H.; PRUSAK, L. Conhecimento empresarial: como as organizações gerenciam o seu capital intelectual. Rio de Janeiro: Campus, 1998.

DE AGUIAR FILHO, A. S.; NASSIF, M. E. O papel dos grupos de apoio e o compartilhamento da informação e do conhecimento nas avaliações das instituições de ensino superior privadas. Perspectivas em Ciência da Informação, v. 21, n. 3, p. 204-221, set. 2016.

DRUCKER, P. Sociedade pós-capitalista. 3. ed. São Paulo: Pioneira, 1993.

E-CONSULTING CORP. A gestão do conhecimento na prática. Revista HSM Management, São Paulo, v. 1, n. 42, p. 53-59, jan./fev. 2004. 
DURST, S.; EDVARDSSON, I. R. Knowledge management in SMEs: a literature review. Journal of Knowledge Management, v. 16 n. 6, p. 879-903, 2012. Disponível em: https://doi.org/10.1108/13673271211276173. Acesso em: 9 abr. 2019.

FAROOQ, R. A conceptual model of knowledge sharing. International Journal of Innovation Science, v. 10, n. 2, p.238-260, 2018.

FRESNEDA, P. S. V.; GOULART, S. M. A experiência brasileira na formulação de uma proposta de política de gestão do conhecimento para a administração pública federal. Câmara dos Deputados, Brasília, 2007.

GOZZI, M. P. O processo de gestão do conhecimento em comunidades virtuais de aprendizagem.

Perspectivas em Gestão \& Conhecimento, João Pessoa, v. 2, n. 2, p. 3-14, jul/dez. 2012.

GROTTO, D. O compartilhamento do conhecimento nas organizações, In: Organizações do conhecimento: infraestrutura, pessoas e tecnologias. São Paulo: Saraiva, 2002. p. 106-119.

HERRERA, C. G. N. El liderazgo en la gestión del conocimento. 2008. Disponível em: https://www.academia.edu/18776696/El_liderazgo_en_la_gesti\%C3\%B3n_del_conocimiento. Acesso em: 15 jul. 2019.

IFSC. Plano de Desenvolvimento Institucional 2015-2019. Florianópolis, 2014. Disponível em: https://www.ifsc.edu.br/pdi. Acesso em: 23 fev. 2018.

Política de Comunicação do IFSC. Florianópolis, 2014. Disponível em: http://www.ifsc.edu.br/documents/23567/0/politica_comunicacao_IFSC_edicao1.pdf. Acesso em: 10 nov. 2018.

Regimento Geral do IFSC. Florianópolis, 2010. Disponível em: http://linkdigital.ifsc.edu.br/files/regimento_geral_atualizado2013.pdf. Acesso em: 30 nov. 2018.

Relatório de Gestão IFSC. Florianópolis, 2017. Disponível em: http://www.ifsc.edu.br/documents/23567/185204/relatorio_gestao_2017.pdf. Acesso em: 30 nov. 2018.

Site Institucional. Disponível em: https://www.ifsc.edu.br/o-ifsc. Acesso em: 15 jul. 2019.

LAKSHMAN, C. Organizational knowledge leadership: an empirical examination of knowledge management by top executive leaders. Leadership \& Organization Development Journal, v. 30, n. 4, p.338-364, 2009.

LEE, K. C.; LEE, S.; KANG, I. W. KMPI: measuring knowledge management performance. Information and Management, v. 42, n. 3, p. 469-482, 2005.

LEITE, F. C. L.; COSTA, S. Repositórios institucionais como ferramentas de gestão do conhecimento científico no ambiente acadêmico. Perspectivas em Ciência da Informação, UFMG, Belo Horizonte, v. 11, n. 2, p. 206 219, maio/ago. 2006.

LIMA, M. A. D. D. S.; ALMEIDA, M. C. P.; LIMA, C. C. A utilização da observação participante e da entrevista semi-estruturada na pesquisa de enfermagem. Revista Gaúcha de Enfermagem, Porto Alegre, v. 20, p. 130142, 1999. Número especial. Disponível em:

https://lume.ufrgs.br/bitstream/handle/10183/23461/000265980.pdf?sequence=1\&isAllowed=y. Acesso em: 19 nov. 2018.

LIAO, C., CHUANG, S. H., TO, P. L. How knowledge management mediates the relationship between environment and organizational structure. Journal of Business Research, v. 64, n. 7, 2011. Disponível em: http://dx.doi.org/10.1016/j.jbusres.2010.08.001. Acesso em: 09 abr. 2019.

LIN, H. Effects of extrinsic and intrinsic motivation on emplouee knowledge sharing intentions. Journal of Information Science, v. 33, n. 2, p. 135-149, 2007. 
LOPEZ, A. P., PEÓN, J. M. M., ORDÁS, C. J. V. Managing knowledge: the link between culture as organizational learning. Journal of Knowledge Management, v. 8 n. 6, p. 93-104, 2004. Disponível em:

https://doi.org/10.1108/13673270410567657. Acesso em: 09 abr. 2019.

MARTINS, A. Compartilhamento do conhecimento entre os professores do departamento de ciências contábeis da Universidade Federal de Santa Catarina. 2007. 49 p. Monografia (Graduação em Ciências Contábeis) - Curso de graduação em Ciências Contábeis da Universidade Federal de Santa Catarina, Florianópolis, 2007.

MAXIMIANO, A. C. A. Introdução à administração. 5. ed. São Paulo: Atlas, 2004.

MINAYO, M. C. et al. Pesquisa social. Petrópolis: Vozes, 1994.

MIRANDA, R. C. D. R. Gestão do conhecimento estratégico: uma proposta de modelo integrado. 2004. 289 f. Tese (Doutorado em Ciência da Informação) - Universidade de Brasília, Brasília, 2004.

MUSSI, C. C.; ANGELONI, M. T. Meios de compartilhamento do conhecimento no contexto de projetos de tecnologia da informação. Revista Estratégia e Negócios, Florianópolis, v. 4, n. 2, p. 30-60, jun./dez. 2011.

NEELY, S. R., COGGBURN, J. D. Incentives for sharing knowledge: a survey of scholarly practices in public affairs and administration. Political Science and Politics, v. 50, n. 2, p. 480-486, 2017.

NONAKA, I.; TAKEUCHI, H. Criação de conhecimento na empresa: como as empresas japonesas geram a dinâmica da inovação. Campus: Rio de Janeiro, 1997.

OCDE. Manual de Oslo: proposta de diretrizes para coleta e interpretação de dados sobre inovação tecnológica, Finep, 1997. Disponível em: http://www.finep.gov.br/images/a-

finep/biblioteca/manual_de_oslo.pdf. Acesso em: 15 jul. 2019.

PROBST, G., RAUB, S. ROMHARDT, K. Gestão do conhecimento: os elementos constitutivos do sucesso. Bookman: Porto Alegre, 2002.

RIZZON, F. TAMIOSSO, L. C. FACHINELLI, A. C. Barreiras ao compartilhamento do conhecimento nas organizações: revisão sistemática de literatura. [2016]. Disponível em:

http://www.ucs.br/etc/conferencias/index.php/mostraucsppga/xvimostrappga/paper/view/4858/1575. Acesso em: 27 nov. 2018.

RODRIGUES, L. C.; MACCARI, É. A. Gestão do conhecimento em instituições de ensino superior. Revista de Negócios, Blumenau, v. 8, n. 2, 2003.

SALIM, J. J. Era digital: o conhecimento em ação. GV Executivo, São Paulo, v. 1, n. 2, p. 55-59, out. 2003. Disponível em: http://dx.doi.org/10.12660/gvexec.v1n2.2003.34804. Acesso em: 08 abr. 2019.

SCHENKEL, M. B. D. C. Compartilhamento do conhecimento científico em instituição estadual de ensino superior: o caso do centro de ciências humanas e da educação da UDESC. 2008. 131 f. Dissertação (Mestrado) - Universidade Federal de Santa Catarina, Centro de Ciências da Educação, Florianópolis, 2008. Disponível em: http://repositorio.ufsc.br/xmlui/handle/123456789/91642. Acesso em: 08 abr. 2019.

SCHONS, C. H. Um estudo do processo de criação do conhecimento nas pequenas empresas de base tecnológica quando do desenvolvimento de novos produtos. 2008. 219 p. Dissertação (Mestrado) Universidade Federal de Santa Catarina, Florianópolis, 2008. Disponível em: http://repositorio.ufsc.br/xmlui/handle/123456789/90850. Acesso em 08 abr. 2019.

SENGE, P. A dança das mudanças. Rio de Janeiro: Campus, 1999. 
SERVIN, G.; DE BRUN, C. ABC of knowledge management. NHS National Library for Health. 2005. Disponível em: https://issuu.com/ddiddo/docs/abc_of_km. Acesso em: 15 jul. 2019.

SORDI, V. F.; NAKAYAMA, M. K.; BINOTTO, E. Compartilhamento de conhecimento nas organizações: um modelo analítico sob a ótica da ação cooperativa. Perspectivas em Gestão \& Conhecimento, João Pessoa, v. 8, n. 1, p. 44-66, 2018.

TAKEUCHI, H.; NONAKA, I. Criação e dialética do conhecimento. In: TAKEUCHI, H.; NONAKA, I. (org.). Gestão do conhecimento. Porto Alegre: Bookman, 2008, p. 17-38.

TEIXEIRA FILHO, J. Gerenciando conhecimento. 2. ed. Rio de Janeiro: SENAC, 2001.

SVEIBY, K. The new organizational wealth: managing and measuring knowledge-based assets. Korean Edition, 2005. Disponível em: https://www.sveiby.com/files/pdf/new-org-wealthpreface.pdf. Acesso em: 08 abr. 2019.

TAYLOR, S. J.; BOGDAN, R. Introducción a lós métodos culitativos de investigación: la búsqueda de significados. Barcelona: Paidós, 1987.

TEODOROSKI, R. C. C. et al. Contribuição do capital intelectual no compartilhamento do conhecimento em uma rede de ensino superior privada. Revista de Gestão e Tecnologia, v. 3, n. 2, p. 105-113, 2013.

TERRA, J.C.C. Gestão do conhecimento: o grande desafio empresarial. 5. ed. Rio de Janeiro: Negócio, 2005.

TONET, H. C.; PAZ, M. D. G. T. Um modelo para o compartilhamento de conhecimento no trabalho. Revista de Administração Contemporânea, v. 10, n. 2, p. 75-94, 2006.

UBON, A.; KIMBLE, C. Knowledge management in online distance education. In: BANKS, S.; GOODYEAR, P.; HODGSON, V.; MCCONNEL, D. (ed.) Proceedings of the 3rd International Conference: Networked Learning 2002. Sheffield: Sheffield University Press, 2002. p. 465-473.

VON KROGH, G. How does social software change knowledge management? toward a strategic research agenda. The Journal of Strategic Information Systems, v. 21, n. 2, p. 154-164, 2012.

WANG, S.; NOE, R. A. Knowledge sharing: a review and directions for future research.

Human Resource Management Review, v. 20, n. 2, p. 115-131, 2010.

YIN, R. K. Pesquisa estudo de caso: desenho e métodos. 2. ed. Porto Alegre: Bookman, 1994.

YUSOF, Z. M. et al. Knowledge sharing in the public sector in Malaysia: a proposed holistic model. Information Development, v. 28, n. 1, p. 43-54, 2012.

ZABOT, J. B. M.; SILVA, L. C. M. Gestão do conhecimento: aprendizagem e tecnologia construindo a inteligência coletiva: São Paulo: Atlas, 2002.

ZHANG X. et al. From e-learning to social-learning: mapping development of studies on social mediasupported knowledge management. Computers in Human Behavior, v. 51, p. 803-811, 2015. 